\title{
MINIREVIEW
}

\section{Cell surface activation of progelatinase A (proMMP-2) and cell migration}

\author{
NAGASE HIDEAKI \\ Department of Biochemistry and Molecular Biology, Uni- \\ versity of Kansas Medical Center, 3901 Rainbow Blvd, \\ Kansas City, KS 66160. E-mail: hnagase@kumc.edu
}

\begin{abstract}
Gelatinase A (MMP-2) is considered to play a critical role in cell migration and invasion. The proteinase is secreted from the cell as an inactive zymogen. In vivo it is postulated that activation of progelationase $\mathrm{A}$ (proMMP-2) takes place on the cell surface mediated by membrane-type matrix metalloproteinases (MT-MMPs). Recent studies have demonstrated that proMMP-2 is recruited to the cell surface by interacting with tissue inhibitor of metalloproteinases-2 (TIMP-2) bound to MT1MMP by forming a ternary complex. Free MT1-MMP closely located to the ternary complex then activates proMMP-2 on the cell surface. MT1-MMP is found in cultured invasive cancer cells at the invadopodia. The MTMMP/TIMP-2/MMP-2 system thus provides localized expression of proteolysis of the extracellular matrix required for cell migration.
\end{abstract}

Key words: GelatinaseA, MT-MMPs, Cell surface activation, TIMP-2, Extracellular matrix.

\section{INTRODUCTION}

Gelatinase A (MMP-2) is a member of the matrix metalloproteinase (MMP) family. MMPs, also called matrixins, are zinc metalloendopeptidases. Because of their abilities to degrade extracellular matrix macromolecules, they are considered to play key roles in many biological processes such as embryo development, morphogenesis, 
angiogenesis, wound healing, and in diseases such as arthritis, atherosclerosis, fibrosis and cancer cell invasion and metastasis[1]. The production of many matrixins is transcriptionally regulated: inflammatory cytokines, growth factors, hormones, cellular transformation and interaction with extracellular matrix components can enhance the transcription of a number of matrixin genes, whereas glucocorticoids, retinoic acids, and ovarian steroids exhibit suppressive effects on some matrixins[2]. In addition, the enzymatic activities are precisely controlled through zymogen activation and inhibition by endogenous specific inhibitor proteins, tissue inhibitors of metalloproteinases (TIMPs) and general protease inhibitors, a -macroglobulins.

A unique feature of MMPs that distinguishes them from other metalloendopeptidases is that the peptide consisting of about 80 amino acids contains a conserved sequence PRCG[V/N]PDV. The cysteinyl residue in this sequence is coordinated with the catalytic $\mathrm{Zn}^{2+}$ of the active site as a fourth ligand. This interaction maintains the inactive zymogen by preventing the association of a water molecule with the $\mathrm{Zn}^{2+}$. Denaturants such as urea and SDS can perturb this interaction and activate the zymogen. A mercurial compound such as $\mathrm{HgCl}_{2}$ and 4-aminophenylmercuric acetate (APMA) also activate most matrixins and this phenomenon is used to characterize matrixins. Many MMP zymogens are also activated by various proteinases. Activation mechanisms of pro-matrixins have been reviewed recently[3]. In most cases, secreted zymogens follow stepwise activation processes: a part of the propeptide is initially removed by a proteolysis or by self-induced cleavage and generates an intermediate, which is then further processed to a fully activated, more stable mature MMP. While most matrixins follow the stepwise activation mechanism, stromelysin 3 (MMP-11) and membrane-type 1 MMP (MT1-MMP or MMP-14) are activated intracellularly by Golgi-associated furin or related serine proteinases. Those zymogens possess the furin recognition sequence $\mathrm{RX}[\mathrm{K} / \mathrm{R}] \mathrm{R}$ at the junction of the $\mathrm{N}$-terminal pro-domain and the catalytic domain. Other MT-MMPs also contain the furinrecognition sequence and they are postulated to be activated intracellularly.

The activation process of proMMP-2 is distinct from those of other MMP zymogens. While it is readily activated by a mercurial compound or SDS in vitro, many proteinases that activate other proMMPs fail to activate this zymogen. It has been reported that interstitial collagenase (MMP-1) and matrilysin (MMP-7) can activate proMMP-2, but the rate of activation is relatively slow, although the activation by MMP-1 is enhanced in the presence of heparin. More likely activators in vivo are considered to be membrane-type MMPs (MT-MMPs) that are expressed on the cell surface[4]. This minireview focuses on the cell surface activation of proMMP-2 and its biological implications.

\section{Cell surface activation of proMMP-2: involvement of TIMP-2}

The activation of proMMP-2 on the cell surface was originally observed when fibroblasts of neoplastic cells were treated with concanavalin A (Con A), a phorbol 
ester, or transforming growth factor $\beta[5,6]$. Indeed, when human fibroblasts are treated with Con A, it enhances the production of MT1-MMP on the cell surface. Currently four MT-MMPs have been identified and MT1-MMP MT2-MMP and MT3-MMP were shown to activate proMMP-2.

Earlier work to investigate the cell surface activation of proMMP-2 used the plasma membrane from the Con-A- or phorbol ester-treated cells. The isolated plasma membrane activates proMMP-2 in a stepwise manner: it initially cleaves the Asn37-Leu38 bond in the middle of the propeptide and then the Asn80-Tyr81 bond by an intermolecular action of the activated MMP-2 intermediate[7]. This activation process requires the interaction of the $\mathrm{C}$-terminal hemopexin-like domain of proMMP-2 and the plasma membrane[8]. The deletion of the C-terminal domain from proMMP-2 or an addition of an excess C-terminal domain prevents the activation[7, 8]. To investigate further the mechanism of proMMP-2 activation, Strongin et al.[9] isolated membrane components from the phorbol ester-treated HT-1080 fibrosarcoma cells that interact with the C-terminal domain of proMMP-2 and identified them as a complex of an activated form of MT1-MMP and TIMP2. An interesting observation was that the activation of proMMP-2 by the plasma membrane was gradually enhanced when a small amount of exogenous TIMP-2 was added to the membrane, but it was inhibited with a larger amount of TIMP-2. The polyclonal antibody raised against TIMP-2 blocked the activation of proMMP2. From those observations they proposed that the activated MT1-MMP on the cell surface serves as a receptor for TIMP-2, which in turn serves as a receptor for proMMP-2. ProMMP-2 and TIMP-2 forms a tight complex through their Cterminal domains. The formation of this ternary complex was, therefore, thought to be critical for activation of proMMP-2 on the cell surface. However, it was not clear how the ternary complex formation of proMMP-2-TIMP-2-MT1-MMP could activate the proMMP-2. Recent studies by Butler et al.[10] have provided further insights into this process. They expressed the recombinant MT1-MMP on the HT1080 fibrosarcoma cells and prepared the TIMP-2-depleted membranes from those cells. The membranes were tested for their ability to activate proMMP-2 in the presence of an exogenously added TIMP-2. When the molar ratios of MT1-MMP to TIMP-2 were $3: 1,2: 1,3: 2$, the activation of proMMP-2 was potentiated compared with the membrane without exogenous TIMP-2. There was no increase in MMP-2 activation when the molar ratio was 7:6, and an excess TIMP-2 to MT1-MMP inhibited the activation of proMMP-2. When TIMP-2 lacking the C-terminal residues 128-194 was added there was no potentiation in proMMP-2 activation, but it was inhibitory. The key region of proMMP-2 that interacts with TIMP-2 was deduced to be residues 563-631 of the C-terminal domain. From those results Butler et al.[10 ] propose that the TIMP-2-MT1-MMP complex acts as a receptor of proMMP-2 and "free" MT1-MMP acts as an activator. Fig 1A depicts this model of activation by MT1-MMP, which suggests that the ratio of free MT1-MMP and the TIMP-2-MT1MMP complex on the cell surface determines the degree of proMMP-2 activation. 


\section{Activation of pro-MT-MMPs}

The presence of the $R X[K / R] R$ sequence between the propeptide and the catalytic domain of MT-MMPs has suggested that those precursors can be activated intracellularly. Pei and Weiss[11] demonstrated that the transmembrane domaindeleted proMT1-MMP was activated intracellularly and the active MT1-MMP was secreted from the cell. This activation is most likely due to a subtilisin-like proprotein converting enzyme. It is therefore postulated that the MT1-MMP expressed on the cell surface is an activated form. In contrast, Cao et al.[12] reported that pro-domain processing was not necessary for MT1-MMP to activate proMMP-2. Cotransfection of COS-1 cells with MT1-MMP cDNA mutated at the furin-recognition site (RRKR $\rightarrow$ ARAA) and proMMP-2 cDNA resulted in activation of MMP-2 to the level similar to that with cells transfected the wild-type MT1-MMP and proMMP-2 cDNAs. Furthermore, co-transfection of the cell with the wild-type MT1-MMP and furin cDNAs did not increase the conversion of pro-MT1-MMP to the active form. It is, however, not known whether both wild-type and mutant MT1-MMPs activate exogenously added proMMP-2 on the cell surface. Studies of Lee et al.[13] reported that fibroblasts cultured in a three-dimensional collagen matrix activated proMMP2 intracellularly. Therefore, it may be speculated that the cells co-transfected with MT1-MMP and proMMP-2 cDNAs may result in activation of proMMP-2 intracellularly without losing the propeptide domain. Clearly, this possibility needs further investigation.

\section{Biological significance of cell surface activation of proMMP-2}

Elevated levels of MMP-2 were found in a number of cancer tissue and the sprouting endothelial cells, suggesting that the enzyme participates in cell migration and proliferation. In immunolocalization studies of MMP-2 in colon cancer the enzyme protein was found in invasive edges of the carcinoma, but MMP-2 mRNA was detected primarily in the stromal cells[14]. These observations emphasize that tumor cells recruit the enzyme produced by surrounding stromal cells and express the proteolytic activity around the tumor cell surface. Three potential mechanisms to recruit MMP-2 to the cell surface have been proposed (see Fig 1).

A first model is the MT1-MMP-TIMP-2 complex as discussed above (Fig 1A). A second model is based on the work by Emmert-Buck et al.[15] who reported the TIMP-2-mediated binding of proMMP-2 to the cell surface of MCF-7 breast adenocarcinoma cells and HT-1080 cells (Fig 1B). TIMP-2 binds directly to those cells through the $\mathrm{N}$-terminal domain of the inhibitor. Since the $\mathrm{kD}$ values of TIMP2 to the receptor of those cells is similar to that of TIMP-2 to the phorbol esteractivated HT-1080 cells[8], this TIMP-2 binding molecule (receptor) may be MT1MMP. 
Fig 1. Modes of cell surface binding of proMMP-2 and its activation.

(A) A proposed activation process of proMMP-2 on the cell surface based on the studies by Strongin et al.[9] and Butler et al.[10]. The MT1-MMP-TIMP-2 complex serves as a cell surface receptor of proMMP-2 (step 1). Free MT1MMP closely located to the ternary complex initiates the activation of proMMP-2 (step 2).

(B) A model of proMMP-2 binding to the cell surface based on the studies by Emmert-Buch et al.[15]. The receptor has not been identified, but it may be MT1-MMP.

(C) A model proposed by Brooks et al.[16]. It is possible that both proMMP-2 and MMP-2 can bind to a v $\beta 3$ integrin. N, N-terminal domain; C, C-terminal domain.
(A)

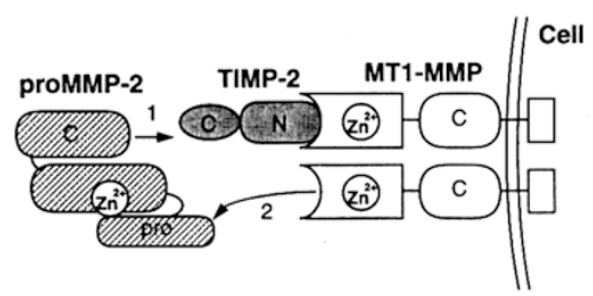

(B)

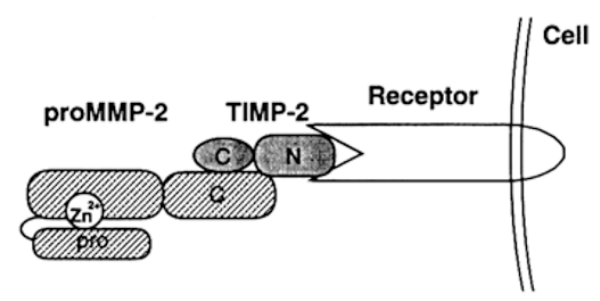

(C)

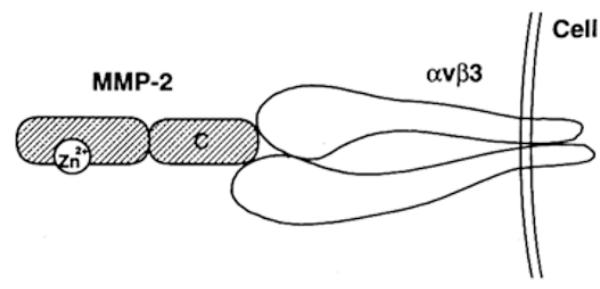

A third model of interaction has been described by Brooks et a1.[16] who reported that MMP-2 binds to a $\beta 33$ integrin (Fig 1C). This interaction is also mediated through the C-terminal hemopexin-like domain of MMP-2. An excess C-terminal domain of MMP-2 inhibits tumor growth by suppressing angiogenesis[17]. This is due to the inhibition of the interaction between MMP-2 and $\alpha \vee \beta 3$ integrin. The recruitment of MMP-2 to the cell surface of endothelial cells and its expression of pericellular proteolytic activity are considered to be critical for neovascularization. In this model of interaction both proMMP-2 and MMP-2 are likely to be bound to $\alpha$ v $\beta 3$. If so, how does the pro-form of MMP-2 become activated? MT1-MMP closely located to $\alpha_{v} \beta 3$ integrin may fill this task. Haas et a1.[18] reported that the network formation of rat microvascular endothelial cells within a type I collagen matrix was associated with an increase of MMP-2 mRNA and MT1-MMP mRNA, and about $50 \%$ of MMP-2 in a three-dimensional collagen matrix was activated, 
whereas only $3.5 \%$ were active in two-dimensional culture, suggesting that activation of MMP-2 by MT1-MMP takes place in endothelial cells undergoing angiogenesis. A synthetic peptidyl-hydroxamate MMP inhibitor blocked the activation of MMP-2 and formation of endothelial cell networks within the collagen gel. The C-terminal domain of MMP-2 would also compete with proMMP-2 activation by MT1-MMP as it interferes with the complex formation between proMMP-2 and TIMP-2 (see Fig 1A).

Thus, the MT-MMP/TIMP-2/MMP-2 system appear to play a pivotal role in both accumulation of proMMP-2 on the cell surface and an expression of local proteolysis of extracellular matrix. Recent work by Nakahara et al.[19] further addresses the importance of the focal expression of MT1-MMP. Their studies demonstrated that transfection of MT1-MMP cDNA into moderately invasive RPMI 7951 human melanoma cells overexpressed MT1-MMP at specialized membrane extensions, invadopodia. Those cells gained the ability to activate soluble and matrix-bound proMMP-2 and degraded and invaded the extracellular matrix. This directional expression of MT1-MMP was dictated by the transmembrane domain and the cytoplasmic domain of MT1-MMP. When those two domains were replaced by the corresponding domains of the interleukin 2 receptor chain, the MT1-MMP failed to be localized at invadopodia. Although this MT1-MMP could activate MMP-2, matrix degradation and invasive activity were not detected in those cells. These results suggest that local concentration of MT1-MMP and the recruitment of proMMP-2 and the its activation are critical events for cellular invasiveness.

\section{Concluding remarks}

The activation of proMMP-2 in vivo is thought to take place on the cell surface. This mechanism allows the initial accumulation of the zymogen on the focal points of cell surface and the subsequent activation of the zymogen. Localized matrix degradation can create new cellular environments. Giannelli et al.[20] recently showed that specific cleavage of the $\mathrm{\gamma} 2$ chain of laminin-5 by MMP-2 exposed a criptic promigratory site on this molecule and triggered cell motility. This emphasizes that proteolysis of extracellular matrix components does not only destroy the matrix but also induce its new biological activities.

Involvement of TIMP-2 for the recruitment of proMMP-2 to the cell surface is unique as TIMP-2 being an endogenous inhibitor of matrixins. We have recently observed that MMP-2 activated by the plasma membrane is largely inhibited by TIMP-2 (Y. Itoh and H. Nagase, unpublished observation), suggesting that TIMP-2 bound to the plasma membrane eventually regulate the activity of MMP-2. However, the detailed molecular interactions of these components have yet to be clarified. A possible intracellular activation mechanism of proMMP-2 also needs further study. Elucidation of the precise molecular mechanisms of these events will then allow us to propose new strategies to regulate cell migration and invasion. 


\section{ACKNOWLEDGEMENT}

This work is supported by NIH grants AR39189 and AR40994.

\section{REFERENCES}

[1] Woessner JF, Jr. The family of matrix metalloproteinases. Ann NY Acad Sci 1994; 732:11-21.

[2] Nagase H. Matrix metalloproteinases. In: Hooper, N. M.ed. Zinc Metalloproteases in Health and Disease. Taylor \& Francis: London 1996:153-204.

[3] Nagase H. Activation mechanisms of matrix metalloproteinases. Biol Chem 1997; 378:151-60.

[4] Sato H, Takino T, Okada Y et al. A matrix metalloproteinase expressed on the surface of invasive tumor cells. Nature 1994; 370:61-5.

[5] Overall CM, Sodek J. Concanavalin A produces a matrix-degradative phenotype in human fibroblasts. J Biol Chem 1990; 265: 21141-21151.

[6] Brown PD, Levy AT, Margulies IMK, Liotta LA, Stetler-Stevenson WG. Independent expression and cellular processing of $\mathrm{Mr} 72,000$ type IV collagenase and interstitial collagenase in human tumorigenic cell lines. Cancer Res 1990; 50:6184-91.

[7] Strongin AY, Marmer BL, Grant GA, Goldberg, GI. Plasma membrane-dependent activation of the $72-\mathrm{kDa}$ type IV collagenase is prevented by complex formation with TIMP-2. J Biol Chem $1993 ; 268: 14033-9$.

[8] Murphy G, Willenbrock F, Ward RV, Cockett MI, Eaton D, Docherty AJP. The C-terminal domain of $72 \mathrm{kDa}$ gelatinase $\mathrm{A}$ is not required for catalysis, but is essential for membrane activation and modulates interactions with tissue inhibitors of metalloproteinases. Biochem $\mathrm{J}$ 1992; 283:637-41.

[9] Strongin AY, Collier I, Bannikov G, Marmer BL, Grant GA, Goldberg GI. Mechanism of cell surface activation of 72-kDa type IV collagenase. J Biol Chem 1995; 270:5331-8.

[10] Butler GS, Butler MJ, Atkinson SJ et al. The TIMP2 membrane type 1 metalloproteinase "receptor" regulates the concentration and efficient activation of progelatinase A. J Biol Chem 1998; 273:871-80.

[11] Pei D, Weiss SJ. Transmembrane-deletion mutants of the membrane-type matrix metalloproteinase1 process progelatinase A and express intrinsic matrix-degrading activity. J Biol Chem 1996; 271:9135-40.

[12] Cao J, Rehemtulla A, Bahou W, Zucker S. Membrane type matrix metalloproteinase 1 activates pro-gelatinase A without furin cleavage of the N-terminal domain. J Biol Chem 1996; 271:3017480.

[13] Lee AY, Akers KT, Collier M, Li L, Eisen AZ, Seltzer JL. Intracellular activation of gelatinase A (72-kDa type IV collagenase) by normal fibroblasts. Proc Natl Acad Sci USA 1997; 94:4424-9.

[14] Poulsom R, Pignatelli M, Stetler-Stevenson WG, Liotta LA, Wright PA, Jeffrey RE. Stromal expression of $72 \mathrm{kDa}$ type IV collagenase (MMP-2) and TIMP-2 mRNAs in colorectal neoplasia. Am J Pathol 1992; 141:389-96.

[15] Emmert-Buck MR, Emonard HP, Corcoran ML, Krutzsch HC, Foidart JM, Stetler-Stevenson WG. Cell surface binding of TIMP-2 and pro-MMP-2/TIMP-2 complex. FEBS Lett 1995; 364:28-32.

[16] Brooks PC, Ströblad S, Sanders LC et al. Localization of matrix metalloproteinase MMP-2 to the surface of invasive cells by interaction with integrin a v $\beta 3$. Cell 1997; 85:683-93.

[17] Brooks PC, Silletti S, von Schalscha TL, Friedlander M, Cheresh DA. Disruption of angiogenesis by PEX, a noncatalytic metalloproteinase fragment with integrin binding activity. Cell 1998; 92:391-400.

[18] Haas TL, Davis SJ, Madri JA. Three-dimensional type I collagen lattices induce coordinate expression of matrix metalloproteinases MT1-MMP and MMP-2 in microvascular endothelial 


\section{Cell surface activation of progelatinase A}

cells. J Biol Chem 1998; 273:3604-10.

[19] Nakahara H, Howard L, Thompson EW et al. Transmembrane/cytoplasmic domain-mediated membrane type 1-matrix metalloprotease docking to invadopodia is required for cell invasion. Proc Natl Acad Sci USA 1997; 94:7959-64.

[20] Giannelli G, Falk-Marzillier J, Schiraldi O, Stetler-Stevenson, WG, Quaranta V. Induction of cell migration by matrix metalloprotease-2 cleavage of laminin-5. Science 1997; 277:225-8. 